\title{
LBM mesoscale modelling of porous media
}

\author{
A. C. M. Sousa ${ }^{1,2} \&$ A. Nabovati ${ }^{1}$ \\ ${ }^{1}$ Department of Mechanical Engineering, University of New Brunswick, \\ Fredericton, NB, Canada \\ ${ }^{2}$ Department of Mechanical Engineering, University of Aveiro, Aveiro, \\ Portugal
}

\begin{abstract}
Permeability is one of the most important bulk properties for the characterization of fluid flow in porous media. However, despite all the considerable body of research work over the past years using experimental, analytical, and numerical approaches, its determination is still a challenge. The methodologies, which have been used to measure, calculate and predict the permeability of different types of porous media, in general, tend to suffer from various levels of limitations in their applicability and, moreover, no general correlation for the permeability is available. Among the different predictive methods for the permeability, numerical pore level fluid flow analyses have been receiving increasing attention in the recent years, due to its robustness and flexibility. In this approach, the viscous fluid flow is directly simulated in the pores of the porous medium with no further modelling required. A simple representation of the pore structure can be in the form of the ordered and random packings of spheres, cylinders or square obstacles. In the present paper, the main objective is to introduce the lattice Boltzmann method (LBM) as a powerful tool for the mesoscopic pore level fluid flow simulation in porous media; two and three-dimensional case studies are presented to demonstrate the capabilities of the mesoscale modelling for porous media fluid flow problems using LBM. To demonstrate an approximation to a reconstructed medium, the fluid flow simulation in a $2 \mathrm{D}$ random arrangement of square obstacles with different aspect ratios is presented. Results of the three-dimensional simulations of the fluid flow in ordered packings of spheres are also reported; the results are in excellent agreement with the available analytical correlation for this configuration.
\end{abstract}

Keywords: pore level analysis, permeability prediction, LBM. 


\section{Introductions}

The objective of the present paper is to review the lattice Boltzmann method (LBM), as a relatively new particle based method for the viscous fluid flow simulation, and to examine its performance when applied to the pore level simulation of fluid flow in porous media.

The investigation of the fluid flow in porous media at pore level enables the researchers to calculate the permeability and other bulk properties of porous media without any further modelling. The importance of the permeability is due to its role in any macroscopic study of fluid flow in porous media; either using the simplest possible model - the Darcy equation [1] or more recent and also more complicated, models (e.g. [2]); it is the permeability that characterizes the medium and it needs to be specified prior to any macro scale modelling. Determination of the permeability is a challenge and despite the considerable body of research work, almost all of the proposed correlations tend to suffer from various levels of restricted applicability; therefore, no general correlation for the permeability is available.

Literature dealing with the permeability prediction is vast and a considerable number of experimental, analytical and numerical studies have proposed different avenues and methodologies to predict the permeability.

The experimental approach has been popular among the researchers for years and still is one of the widely used methods to study the porous media flow (e.g. [3-9]). Jackson and James [10] and Skartsis et al. [11] have collected a comprehensive bibliography of the experimental and numerical research work conducted in this field.

Most of the analytical efforts to predict the permeability consider the porous media to be constructed as ordered arrangements of objects of simple geometrical shape, and the flow around them is described by a particular mathematical formulation. Emersleben in 1925, as reported in [11], was the first one who studied the flow parallel to square arrays of cylinders by solving the Navier Stokes equations. Larson and Higdon [12, 13] numerically studied the axial and transverse flow between square and hexagonal arrays of cylinders using the boundary integral method. Keller [14], Sangani and Acrivos [15], Drummond and Tahir [16], Higdon and Ford [17] have also considered the porous media to be in the form of ordered packing of cylinders with different arrangements.

Flow in different three-dimensional lattices of spheres was also analytically solved by Hasimoto [18] and Sangani and Acrivos [19]. The main shortcoming related to these studies is the lack of generality of the proposed correlations due to the simplifications introduced in the equations to make them amenable to the particular analytical method used.

An advanced method for the permeability prediction deals with the numerical simulation of the fluid flow in the inter-grain regions and inside the pores of the porous medium by solving either the Navier Stokes equations or their equivalent forms, such as, the Lattice Boltzmann equation. Then, the permeability of the 
medium can be calculated using the Darcy law [1] by averaging the local velocities over the flow, namely:

$$
\langle\vec{u}\rangle=-\frac{\mathbf{K}}{\mu} \frac{d p}{d \vec{x}}
$$

where $\langle\vec{u}\rangle, \mathbf{K}, \mu$ and $d p / d \vec{x}$ are the volume averaged velocity, permeability tensor, fluid viscosity and the applied pressure gradient.

The very first requirement in pore level analysis is to determine the grain structure and the pore geometry of the porous medium. The computed tomography (CT) imaging technique has had some success in the determination of pore structures [20, 21]; but, in general, the application of experimental imaging methods is costly and has its own limitations. An alternate approach is to reconstruct the porous medium by arrangements of the geometrical volumes, either orderly or randomly. Due to its simplicity and ability to reconstruct media similar to the actual structures, this method has been popular among the researchers in this field.

Numerically solving the fluid flow in the inter-grain voids, removes the limitation of considering simple geometric shapes for the grains; however, it still demands very high computational resources to resolve the inter-grain structure accurately, which for years had limited the applicability of the numerical methods to very simple geometries [22, 23]. In recent years, with the development of advanced numerical methods and increased availability of computational resources, more complex pore level geometries could be implemented. A particular difficulty related to the numerical pore level flow simulation approach is the limited applicability of the Navier Stokes equations to the micro flows, when local high velocities occur at the narrow pores, which is more likely to happen in the low porosity structures with random pore structure. The treatment of complex solid wall boundary conditions is also a cumbersome task when Navier Stokes equations are used. To overcome these two potential shortcomings of the pore level analysis, some novel methods for the fluid flow simulation have been proposed, namely: the Lattice Boltzmann Method [24, 25] and the Smoothed Particle Hydrodynamic [26,27]. The main unresolved difficulty associated with the SPH method is the exponential computational cost with the increasing number of particles, which nearly precludes performing physically meaningful three-dimensional simulations. Similar problem was encountered with the early versions of the LBM introduced first by McNamara and Zanetti [28], but soon after it was removed by Qian et al. [29] and since then the LBM has been applied successfully to different problems of flow in porous media [30-34].

The present work addresses the LBM pore level fluid flow simulation in porous media - one of the earliest and most important fields of research to which the LBM has been successfully applied. Simulation results for the flow in two and three-dimensional reconstructed porous media are presented and the predicted permeability is compared against the available correlations to demonstrate the LBM capabilities. 


\section{Lattice Boltzmann method}

Following the notation of Geller et al. [35], the form of the lattice Boltzmann equation (LBE) used in the present study can be regarded as the first order explicit upwind finite difference discretization of the discrete Boltzmann equation, as follows:

$$
f_{i}\left(\vec{x}+\vec{e}_{i} \delta t, t+\delta t\right)-f_{i}(\vec{x}, t)=\Omega_{i}\left(f_{i}(\vec{x}, t)\right) \quad i \in\{0, b\}
$$

where $f_{i}$ is the particle distribution function, $\vec{x}$ and $\mathrm{t}$ are the spatial coordinate and time, $b+1$ is the number of the discrete lattice speeds (in the present work, nine-speed lattice in 2D and 15-speed lattice in 3D simulations were employed), and $\Omega_{i}$ is the collision operator, which for the single relaxation time LBE is defined as follows:

$$
\Omega_{i}=-\frac{\delta t}{\tau}\left(f_{i}(\vec{x}, t)-f_{i}^{e q}(\vec{x}, t)\right) \quad i \in\{0, b\}
$$

where $\tau$ is the lattice relaxation time and $\delta t$ is the lattice time step, related to the lattice length scale, $\delta x$, as $c=\delta x / \delta t$; in most of the LBM simulations on a uniform lattice, including the present work, $\delta x$ and $\delta t$ are considered to be equal to one for simplicity. The equilibrium distribution function is the solution of the Boltzmann equation in the absence of any external force and zero spatial and temporal gradients.

Depending on the type of the lattice and number of the speeds, the particle distribution function has $\mathrm{b}+1$ components, in the form of

$$
f_{i}(\vec{x}, t)=\left[f_{0}(\vec{x}, t), f_{1}(\vec{x}, t), \ldots, f_{b}(\vec{x}, t)\right]^{\mathrm{T}}
$$

Having calculated the distribution function on each lattice site using eqn (2), the average flow hydrodynamic parameters can be calculated as follows:

$$
\begin{array}{r}
\rho=\sum_{i=0}^{b} f_{i}=\sum_{i=0}^{b} f_{i}^{e q} \\
\rho \vec{u}=\sum_{i=0}^{b} f_{i} \vec{e}_{i}=\sum_{i=0}^{b} f_{i}^{e q} \vec{e}_{i}
\end{array}
$$

The kinematic viscosity of the fluid is calculated as a function of the relaxation time and the lattice time step as:

$$
v=\frac{c^{2}}{3}\left(\tau-\frac{\delta t}{2}\right)
$$

and the relation between the lattice sound speed and the pressure is as follows

$$
p=\rho c_{s}^{2}
$$

where $c_{s}$ is the lattice sound speed and is equal to $c / \sqrt{3}$. One of the main advantages of using the LBM is the easy implementation of the solid wall boundary condition, which in the present study was implemented by using the 
simple bounceback method [24] for both the two and three-dimensional simulations. The applied pressure gradient was treated as a uniform body force, which was added to the right hand side of eqn (2) at each time step on all the fluid nodes.

\section{Results}

A simple representation of the pore structure can be in the form of the ordered or random packings of spheres, cylinders or square obstacles. As a first approximation for a reconstructed medium, the fluid flow in $2 \mathrm{D}$ random arrangements of square obstacles of different aspect ratio was simulated by the present authors [34]. Predictions of the permeability were conducted and they were in good agreement with the available correlations. The effect of the obstacles' aspect ratio on the permeability was also studied.

For the three-dimensional study of the pore level flow, the packing of spheres is the most widely used structure; the reason is that the analytical flow solution around a sphere has been available in the literature for some time; also the packing of spheres resembles many of the real world porous media structures. Sangani and Acrivos [19] reported analytical correlations for the permeability of three different arrangements of spheres of constant radius - Simple Cubic (SC), Body Centred Cubic (BCC), and Face Centred Cubic (FCC) - in terms of series expansion solution of Stokes flow, as follows:

$$
K=\frac{L^{3}}{6 \beta \pi a C_{d}}
$$

where $K, a$ and $L$ are the permeability, sphere radius and unit cube side length; $\beta$ is the number of spheres in a cube of size $L^{3}$, which is equal to 1,2 and 4 for the SC, BCC and FCC arrangements, respectively. $C_{d}$ is calculated as follows:

$$
C_{d}=\sum_{n=0}^{30} \alpha_{n} \chi^{n}
$$

where the terms $\alpha_{n}$ are the series coefficients tabulated for each arrangement [19] and $\chi$ is a function of the solid volume fraction of the structure, $c$, as follows:

$$
\chi=\left(c / c_{\max }\right)^{1 / 3}
$$

$c_{\max }$ is the maximum solid fraction for each arrangement, determined by the maximum sphere radius in a cube of side length of $\mathrm{L}$ for which spheres are in contact without overlapping. $c_{\max }$ is equal to $\pi / 6, \sqrt{3} \pi / 8$ and $\sqrt{2} \pi / 6$ for the SC, BCC and FCC structures, respectively.

Figure 1 shows the velocity magnitude contours and the velocity vectors in a BCC structure; cube side length $(L)$ is 51 lattice units and the spheres' radius $(a)$ is 16 lattice units, which yields a porosity of 0.87 . 
Figure 2 presents the comparison between the predicted permeability and the correlation of Sangani and Acrivos [19] for three different arrangements of spheres. As it can be seen, the agreement between the predicted values by the numerical simulation and the analytical correlation is excellent. For the low porosities, as was stated by Sangani and Acrivos [19], the series did not converge and the analytical solution is not accurate; which justifies the discrepancy between the numerical simulation and the analytical correlation.
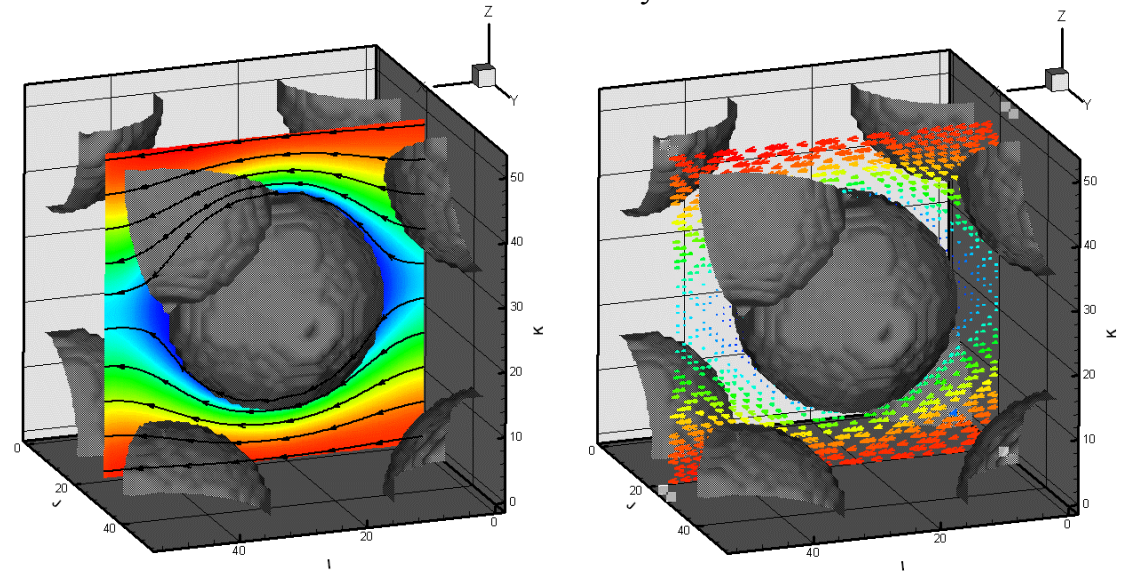

Figure 1: Velocity magnitude contours and the velocity vectors in a BCC structure; $L=51$ and $a=6$ lattice units, which yields a porosity of 0.87 .

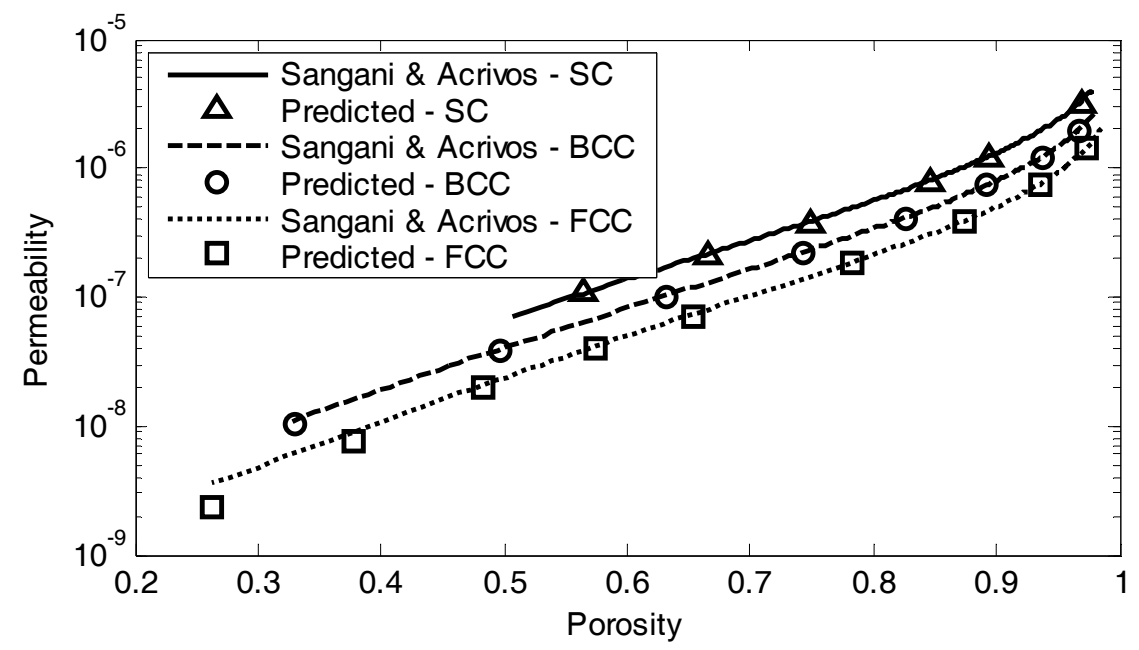

Figure 2: Comparison of the predicted permeability against the analytical correlation of Sangani and Acrivos [19] for three different arrangements of spheres, namely: SC, BCC, and FCC. 
To change the porosity of each arrangement, size of the representative volume, $L$, was kept constant and the porosity was changed by varying the spheres' radius. As it is shown in figure 3, normalizing the predicted permeability by the square of the spheres' radius, $a$, for a constant value of $L$, causes the predicted values of the permeability for all the three different structures fall on the same curve, and eliminates the effect of the sphere arrangement when plotted as a function of the medium porosity. The normalized predicted permeability can be correlated as a function of the porosity, $\varphi$, as follows:

$$
\begin{gathered}
\frac{K}{a^{2}}=\exp \left(a_{3} \Phi^{3}+a_{2} \Phi^{2}+a_{1} \Phi+a_{0}\right) \\
\Phi=\ln \left(\frac{\varphi}{1-\varphi}\right)
\end{gathered}
$$

where the fitting parameters, $a_{3}, a_{2}, a_{1}, a_{0}$ are calculated to be equal to 0.0118 , $-0.2093,2.2648$, and -4.6254 , respectively. The correlation above is valid for all the three arrangements, namely $\mathrm{SC}, \mathrm{BCC}, \mathrm{FCC}$, and for the entire range of the accessible porosity.

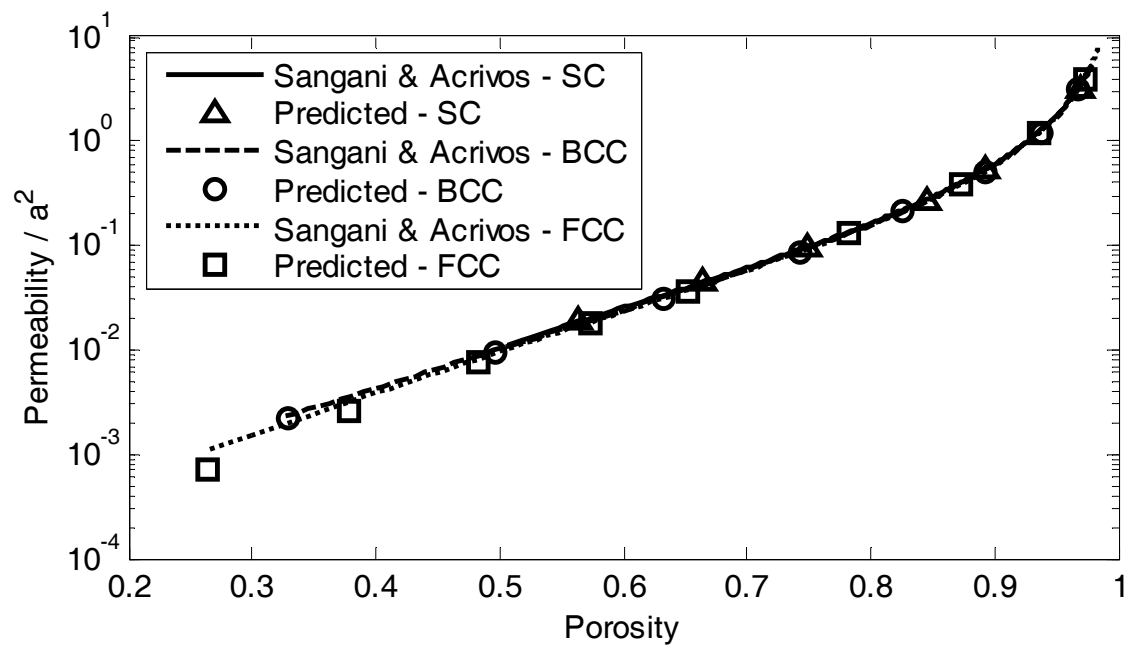

Figure 3: Normalized predicted permeability by the sphere radius versus porosity for three different arrangements of spheres, namely SC, $\mathrm{BCC}$, and $\mathrm{FCC}$.

\section{Conclusion}

The mesoscopic nature of the lattice Boltzmann method along with the implementation of the solid wall boundary condition make this approach a powerful tool for the pore level fluid flow simulation in porous media. Due to its 
mesoscopic nature, the LBM enables its use in both the macroscopic and microscopic flow simulations, where the conventional CFD methods based on the Navier Stokes equations require some modifications. Using the LBM for the pore level fluid flow simulations in porous media requires no further modelling and the bulk properties of the porous can be calculated as the outcome of the simulation.

The demonstration of the LBM capabilities was first performed in a previous publication, where the fluid flow was simulated in a two dimensional random arrangement of square obstacles and the effect of the aspect ratio on the permeability was studied. In the present work, to further demonstrate and test LBM, three-dimensional simulations were performed for the fluid flow in a three-dimensional ordered packing of spheres with three different arrangements, namely: SC, BCC, and FCC. Keeping the size of the representative volume constant, the porosity of the reconstructed medium was changed by varying the spheres' radius. The predicted permeability was in excellent agreement with the available analytical correlation for the entire range of the accessible porosity. Normalizing the permeability by the square of the spheres' radius causes all the predicted values to collapse on a single curve and eliminates the effect of the sphere arrangement on the permeability. A correlation for the normalized permeability as a function of the porosity was proposed, which is valid for the entire range of the porosity and the all the three arrangements.

\section{Acknowledgements}

The authors acknowledge the support received from NSERC (Natural Sciences and Engineering Research Council of Canada) Discovery Grant 12875 (ACMS) and from the Foundation for Science and Technology (FCT, Portugal) through the research grant POCTI/EME/59728/2004 (ACMS).

\section{References}

[1] Kaviany, M., Principles of Heat Transfer in Porous Media, Springer Verlag: New York, 1991.

[2] Vafai, K. \& Thiyagaraja, R., Analysis of flow and heat transfer at the interface region of a porous medium", International Journal of Heat and Mass Transfer, 30(7), pp. 1391-1405, 1987.

[3] Tomadakis, M.M. \& Robertson, T.J., Viscous permeability of random fiber structures: Comparison of electrical and diffusional estimates with experimental and analytical results, Journal of Composite Materials, 9, pp. 163-188, 2005.

[4] Loosveldt, H., Lafhaj, Z. \& Skoczylas, F., Experimental study of gas and liquid permeability of a mortar, Cement and Concrete Research, 32(9), pp. 1357-1363, 2002.

[5] Seguin, D., Montillet, A. \& Comiti, J., Experimental characterization of flow regimes in various porous media - I: Limit of laminar flow regime, Chemical Engineering Science, 53(21), pp. 3751-3761, 1998. 
[6] Rahli, O., Tadrist, L. \& Miscevic, M., Experimental analysis of fibrous porous media permeability, AICHE Journal, 42(12), pp. 3547-3549, 1996.

[7] Rashidi, M., Peurrung, L., Tompson, A.F.B. \& Kulp, T.J., Experimental analysis of pore-scale flow and transport in porous media, Advances in Water Resources, 19(3), pp. 163-180, 1996.

[8] Comiti, J. \& Renaud, M., A new model for determining mean structure parameters of fixed-beds from pressure-drop measurements - application to beds packed with parallelepipedal particles, Chemical Engineering Science, 44(7), pp. 1539-1545, 1989.

[9] Williams, J.G., Morris C.E.M. \& Ennis, B.C., Liquid Flow through Aligned Fiber Beds, Polymer Engineering and Science, 14(6), pp. 413-419, 1974.

[10] Jackson, G.W. \& James, D.F., The Permeability of Fibrous Porous-Media, Canadian Journal of Chemical Engineering, 64(3), pp. 364-374, 1986.

[11] Skartsis, L., Khomami, B. \& Kardos, J.L., Resin Flow Through Fiber Beds During Composite Manufacturing Processes. Part II: Numerical and Experimental Studies of Newtonian Flow Through Ideal and Actual Fiber Beds, Polymer Engineering and Science, 32(4), pp. 231-239, 1992.

[12] Larson, R.E. \& Higdon, J.J.L., Microscopic Flow near the Surface of TwoDimensional Porous-Media. Part 1. Axial Flow, Journal of Fluid Mechanics, 166, pp. 449-472, 1986.

[13] Larson, R.E. \& Higdon, J.J.L., Microscopic Flow near the Surface of TwoDimensional Porous-Media. Part 2. Transverse Flow, Journal of Fluid Mechanics, 178, pp. 119-136, 1987.

[14] Keller, J.B., Viscous flow through a grating or lattice of cylinders, Journal of Fluid Mechanics, 18, pp. 94-96, 1964.

[15] Sangani, A.S. \& Acrivos, A., Slow flow past periodic arrays of cylinders with application to heat transfer, International Journal of Multiphase Flow, 8(3), pp. 193-206, 1982.

[16] Drummond, J.E. \& Tahir, M.I., Laminar viscous flow through regular arrays of parallel solid cylinders, International Journal of Multiphase Flow, 10(5), pp. 515-540, 1984.

[17] Higdon, J.J.L. \& Ford, G.D., Permeability of three-dimensional models of fibrous porous media, Journal of Fluid Mechanics, 308, pp. 341-361, 1996.

[18] Hasimoto, H., On the periodic fundamental solutions of the stokes equations and their application to viscous flow past a cubic array of spheres, Journal of Fluid Mechanics, 5, pp. 317-328, 1959.

[19] Sangani, A.S. \& Acrivos, A., Slow flow through a periodic array of spheres, International Journal of Multiphase Flow, 8(4), pp. 343-360, 1982.

[20] Heijs, A.W.J. \& Lowe, C.P., Numerical evaluation of the permeability and the Kozeny constant for two types of porous media, Physical Review E, 51, pp. 4346-4353, 1995.

[21] Hazlett, R.D., Simulation of Capillary-Dominated Displacements in Microtomographic Images of Reservoir Rocks, Transport in Porous Media, 20, pp. 21-35, 1995. 
[22] Sahraoui, M. \& Kaviany, M., Slip and no slip velocity boundary conditions at interface of porous, plain media, International Journal of Heat and Mass Transfer, 35(4), pp. 927-943, 1992.

[23] Nakayama, A., Kuwahara, F., Umemoto, T. \& Hayashi, T., Heat and Fluid Flow Within an anisotropic porous medium, Journal of Heat Transfer, 124, pp. 746-753, 2002.

[24] Succi, S., The Lattice Boltzmann Equation - For Fluid Dynamics and Beyond, Oxford University Press: Oxford, 2001.

[25] Chen, S. \& Doolen, G.D., Lattice Boltzmann method for fluid flows, Annual Review of Fluid Mechanics, 30, pp. 329-364, 1998.

[26] Jiang, F., Oliveira, M.S.A. \& Sousa, A.C.M., Mesoscale SPH modeling of fluid flow in isotropic porous media, Computer Physics Communications, 176 (7), pp. 471-480, 2007.

[27] Jiang, F. \& Sousa, A.C.M., Smoothed Particle Hydrodynamics Modeling of Transverse Flow in Randomly Aligned Fibrous Porous Media, Transport in Porous Media, DOI: 10.1007/s11242-008-9206-z, available online since February 2008.

[28] McNamara, G.R. \& Zanetti, G., Use of the Boltzmann Equation to Simulate Lattice-Gas Automata, Physical Review Letters, 61(20), pp. 2332-2335, 1988.

[29] Qian, Y.H., d'Humieres, D. \& Lallemand, P., Lattice BGK model for Navier-Stokes equation, Europhysics Letters, 17(6BIS), pp. 479-484, 1992.

[30] Succi, S., Foti, E. \& Higuera, F., Three-dimensional flows in Complex Geometries with the Lattice Boltzmann Method, Europhysics Letters, 10(5), pp. 433-438, 1989.

[31] Cancelliere, A., Chang, C., Foti, E., Rothman, D.H. \& Succi, S., The permeability of a random medium: Comparison of simulation with theory, Physics of Fluids A, 2(12), pp. 2085-2088, 1990.

[32] Koponen, A., Kataja, M. \& Timonen, J., Permeability and effective porosity of porous media, Physical Review E, 56(3), pp. 3319-3325, 1997.

[33] Nabovati, A. \& Sousa, A.C.M., Fluid Flow Simulation at the Open-Porous interface Using Lattice Boltzmann Method, International Journal for Numerical Methods in Fluids, 56(8), pp. 1449 - 1456, 2008.

[34] Nabovati, A. \& Sousa, A.C.M., Fluid Flow Simulation in Random Porous Media at Pore Level using the Lattice Boltzmann Method, Journal of Engineering Science and Technology, 2(3), pp. 226- 237, 2007.

[35] Geller, S., Krafczyk, M., Tolke, J., Turek, S. \& Hron, J., Benchmark computations based on lattice-Boltzmann, finite element and finite volume methods for laminar flows, Computers and Fluids, 35, pp. 888-897, 2006. 